\title{
Peculiarities of luminescence decay of Ce:LaF 3 nanoparticles depending on conditions of hydrothermal treatment
}

\author{
Elena Lukinova*, Eduard Madirov, Maxim Pudovkin, Daria Koryakovtseva, \\ Stella Korableva, Alexey Nizamutdinov, and Vadim Semashko \\ Kazan Federal University, Institute of Physics, 18 Kremlevskaja str., Kazan, 420008 Russia
}

\begin{abstract}
Luminescence decay properties of crystalline $\mathrm{LaF}_{3}$ nanoparticles containing $12 \%$ of $\mathrm{Ce}^{3+}$ ions collected at different stages of microwave hydrothermal treatment were studied. The microwave treatment has led to improvement of surface perturbed sites luminescence together with reducing PL decay time of regular sites apparently due to higher surface/volume ratio.
\end{abstract}

\section{Introduction}

Fluoride crystalline nanoparticles and $\mathrm{LaF}_{3}$ particularly doped with $\mathrm{RE}$ ions exhibit prospective luminescent properties. Due to low-energy phonons these materials provide advantages in up-conversion and photosensitizing demanding applications [1,2] and open novel applications for quantum electronics and photonics [3].

The variation of synthesis conditions which leads changes in energy transfer processes between impurities and influences the luminescence quenching factors [2] opens the way to manage characteristics of materials. Aim of this work is to study luminescence decay properties of $\mathrm{Ce}^{3+}$ ions in $\mathrm{LaF}_{3}$ nanoparticles after microwave hydrothermal treatment.

\section{Experimental}

The nanoparticles of $\mathrm{LaF}_{3}$ doped with $12 \%$ of $\mathrm{Ce} 3+$ ions were synthesized via coprecipitation method [3-5] and subsequent modification of prepared nanoparticles using the microwave-assisted hydrothermal treatment was performed [6]. Four samples collected at different stages of fabrication were investigated. Namely N1 sample was obtained right after precipitation procedure. The N2 sample was collected right after the $\mathrm{pH}$ was adjusted to 5-6 by adding $25 \%$ solution of ammonium hydrate and then stirred with heating for 2 hours. The remaining suspension was placed into the microwave oven for 30 minutes (sample N3) and 180 minutes (sample N4). All samples were washed with distillate water and separated from the suspension by centrifugation. The resulting product was dried at $60^{\circ} \mathrm{C}$ for $12 \mathrm{~h}$.

\footnotetext{
*Corresponding author: elena.v.lukinova@gmail.com
} 


\section{Results and discussion}

Luminescence (PL) spectrum of Ce:LaF $\mathrm{Lample}_{3}$ s1 registered under $266 \mathrm{~nm}$ excitation (see Fig. 1a) appeared to be characteristic for all samples and coinciding with literature [2].
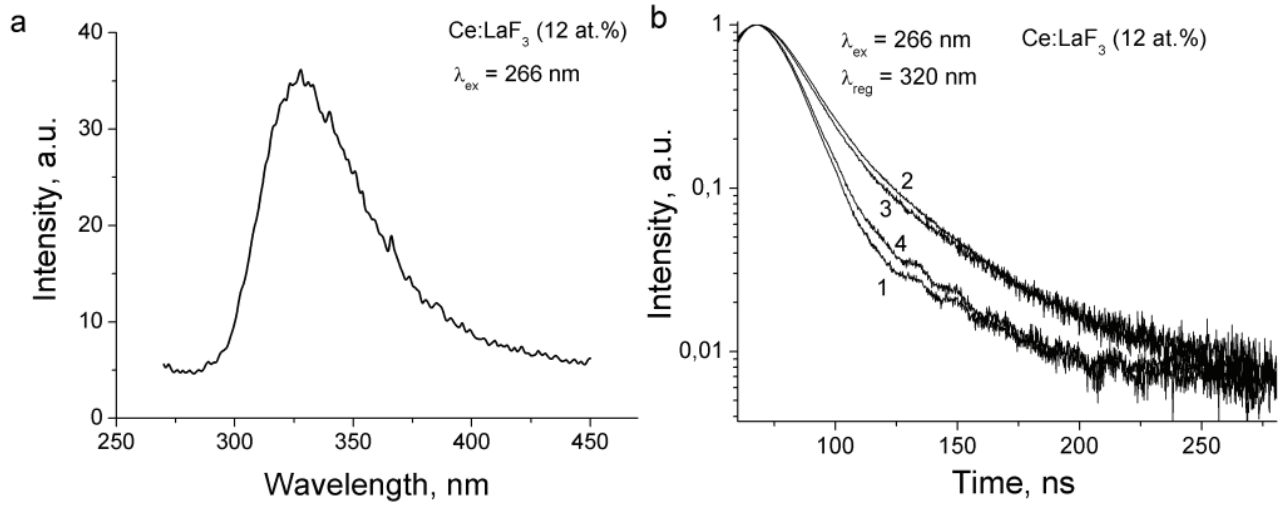

Fig. 1. Room temperature PL spectrum (a) and PL decay curves (b) of $\mathrm{LaF}_{3}: \mathrm{Ce}(12 \%)$ nanoparticles dried samples, 1 - N1 sample, 2 - N2 sample, 3 - N3 sample, 4 - N4 sample.

The decay curves of PL detected at $320 \mathrm{~nm}$ of all four samples of Ce: $\mathrm{LaF}_{3}$ nanoparticles show fast and slow components (see Fig. 1b) determined by regular sites and perturbed sites relaxation respectively [2]. Results of the fitting the decays by two-exponential dependence are presented in Table 1. It is shown that both fast and slow components exhibit changes with synthesis procedure.

Table 1. Luminescence decay components of $\mathrm{Ce}_{\mathrm{LaF}} \mathrm{La}_{3}$ nanoparticles samples.

\begin{tabular}{|c|c|c|c|c|}
\hline & $\mathrm{LaF}_{3}: \mathrm{Ce}(\mathrm{N} 1)$ & $\mathrm{LaF}_{3}: \mathrm{Ce}(\mathrm{N} 2)$ & $\mathrm{LaF}_{3}: \mathrm{Ce}(\mathrm{N} 3)$ & $\mathrm{LaF}_{3}: \mathrm{Ce}(\mathrm{N} 4)$ \\
\hline $\mathrm{t} 1, \mathrm{~ns}$ & $55.6 \pm 0.5$ & $36.6 \pm 0.5$ & $38.2 \pm 0.5$ & $43.1 \pm 0.5$ \\
\hline $\mathrm{t} 2, \mathrm{~ns}$ & $8.1 \pm 0.5$ & $9.8 \pm 0.5$ & $10.1 \pm 0.5$ & $7.9 \pm 0.5$ \\
\hline
\end{tabular}

It can be seen that stabilization of $\mathrm{pH}$ drops PL decay time for perturbed state and this can affect energy transfer to sensitizers. Also it is shown that microwave treatment somehow improves this effect together with reducing PL decay time of regular sites apparently due to higher surface/volume ratio.

The work was supported by the subsidy of the Russian Government (agreement No.02.A03.21.0002) to support the Program of Competitive Growth of Kazan Federal University and for the subsidy allocated to Kazan Federal University for the state assignment in the sphere of scientific activities [3.1156.2017/4.6].

\section{References}

[1] K. Binnemans, Chemical Reviews 109, 4283 (2009)

[2] D. R. Cooper et al., Phys. Chem. Chem. Phys. 16, 12441 (2014)

[3] M. S. Pudovkin et al., J. of Nanomaterials 2017, 3108586 (2017)

[4] F. Wang, Y. Zhang, X. Fanc, M. Wang, J. Mater. Chem. 16, 1031 (2006)

[5] J. Shao et al., J. of Solid State Chem. 49, 199 (2017)

[6] E. M. Alakshin et al., J. of Nanomaterials 2016, 7148307 (2016) 